\title{
Association analysis between mortality and ionizing radiation exposure in French uranium workers
}

\author{
Ségolène Bouet ${ }^{1}$, Estelle Davesne ${ }^{2}$, Eric Samson ${ }^{1}$, Iris Jovanovic ${ }^{3}$, Eric Blanchardon ${ }^{2}$, \\ Cécile Challeton - de Vathaire ${ }^{2}$, David B Richardson ${ }^{4}$, Klervi Leuraud ${ }^{1}$, Dominique \\ Laurier $^{5}$, and Olivier Laurent ${ }^{1 *}$ \\ 1 Institut de Radioprotection et de Sûreté Nucléaire (IRSN), Laboratoire d'Epidémiologie des \\ rayonnements ionisants (PSE-SANTE/SESANE/LEPID), Fontenay-aux-Roses, France \\ 2 Institut de Radioprotection et de Sûreté Nucléaire (IRSN), Laboratoire d'Evaluation de la Dose \\ Interne (PSE-SANTE/SDOS/LEDI), Fontenay-aux-Roses, France \\ 3 Amplexor, Montigny-le-Bretonneux, France \\ 4 University of North Carolina, Chapel Hill, USA \\ 5 Institut de Radioprotection et de Sûreté Nucléaire (IRSN), PSE-SANTE/SESANE, Fontenay-aux- \\ Roses, France
}

To investigate associations between mortality and chronic internal and external exposure to ionizing radiation in a cohort of nuclear workers with potential for internal exposure to uranium.

Workers employed for at least six months in five plants involved in the French nuclear fuel cycle were included and followed up for mortality between 1968 and 2013. Causespecific standardized mortality ratios were calculated for all workers. Internal and external absorbed doses were reconstructed for each worker with computerized information. Analyses of associations between radiation dose and cause-specific mortality were conducted using Poisson regression.

The cohort includes 4541 workers. At the end of the follow-up, 838 workers were deceased and 28 lost to follow-up. A healthy worker effect was observed. The risk of prostate cancer mortality was significantly higher for workers exposed to cumulative external dose higher than $50 \mathrm{mGy}$ as compared to non-exposed, but this association became non-significant after adjustment for smoking. Associations between mortality and internal dose showed no consistent pattern. Except for prostate cancer mortality, adjustments for smoking and other risk factors rarely documented in cohorts of radiation workers had no marked influence on findings.

It is the first time in France that such a study was conducted in a cohort of uranium workers with a complete reconstruction of internal dose. Results should be considered as preliminary and interpreted with caution because of the limited size of the cohort and significant sources of uncertainty, notably on dose assessment. Future steps of this study will allow overcoming these limitations.

\footnotetext{
*Corresponding author: olivier.laurent@irsn.fr
} 